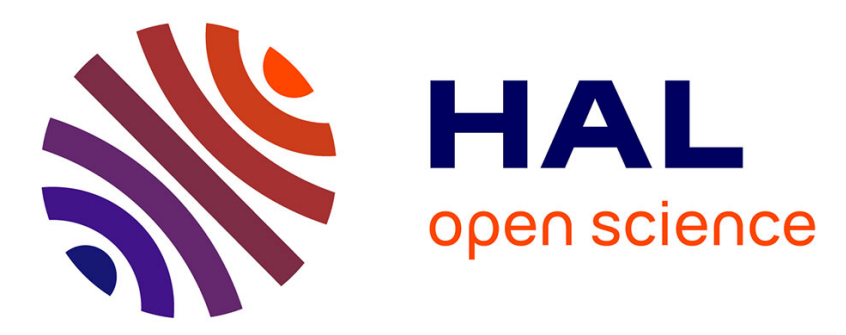

\title{
On the multistream approach of relativistic Weibel instability. III. Comparison with full-kinetic Vlasov simulations
}

Alain Ghizzo

\section{- To cite this version:}

Alain Ghizzo. On the multistream approach of relativistic Weibel instability. III. Comparison with fullkinetic Vlasov simulations. Physics of Plasmas, 2013, 20 (8), pp.82111 - 82111. 10.1063/1.4817752 . hal-01287084

\section{HAL Id: hal-01287084 \\ https://hal.science/hal-01287084}

Submitted on 20 Apr 2018

HAL is a multi-disciplinary open access archive for the deposit and dissemination of scientific research documents, whether they are published or not. The documents may come from teaching and research institutions in France or abroad, or from public or private research centers.
L'archive ouverte pluridisciplinaire HAL, est destinée au dépôt et à la diffusion de documents scientifiques de niveau recherche, publiés ou non, émanant des établissements d'enseignement et de recherche français ou étrangers, des laboratoires publics ou privés. 


\section{On the multistream approach of relativistic Weibel instability. III. Comparison with full- kinetic Vlasov simulations}
A. Ghizzo

Citation: Physics of Plasmas 20, 082111 (2013); doi: 10.1063/1.4817752

View online: https://doi.org/10.1063/1.4817752

View Table of Contents: http://aip.scitation.org/toc/php/20/8

Published by the American Institute of Physics

\section{Articles you may be interested in}

On the multistream approach of relativistic Weibel instability. I. Linear analysis and specific illustrations

Physics of Plasmas 20, 082109 (2013); 10.1063/1.4817750

On the multistream approach of relativistic Weibel instability. II. Bernstein-Greene-Kruskal-type waves in magnetic trapping

Physics of Plasmas 20, 082110 (2013); 10.1063/1.4817751

Multidimensional electron beam-plasma instabilities in the relativistic regime

Physics of Plasmas 17, 120501 (2010); 10.1063/1.3514586

Electron streams formation and secondary two stream instability onset in the post-saturation regime of the classical Weibel instability

Physics of Plasmas 18, 052104 (2011); 10.1063/1.3587089

Electron holes in phase space: What they are and why they matter

Physics of Plasmas 24, 055601 (2017); 10.1063/1.4976854

Nonlinear saturation of relativistic Weibel instability driven by thermal anisotropy

Physics of Plasmas 16, 082103 (2009); 10.1063/1.3172941

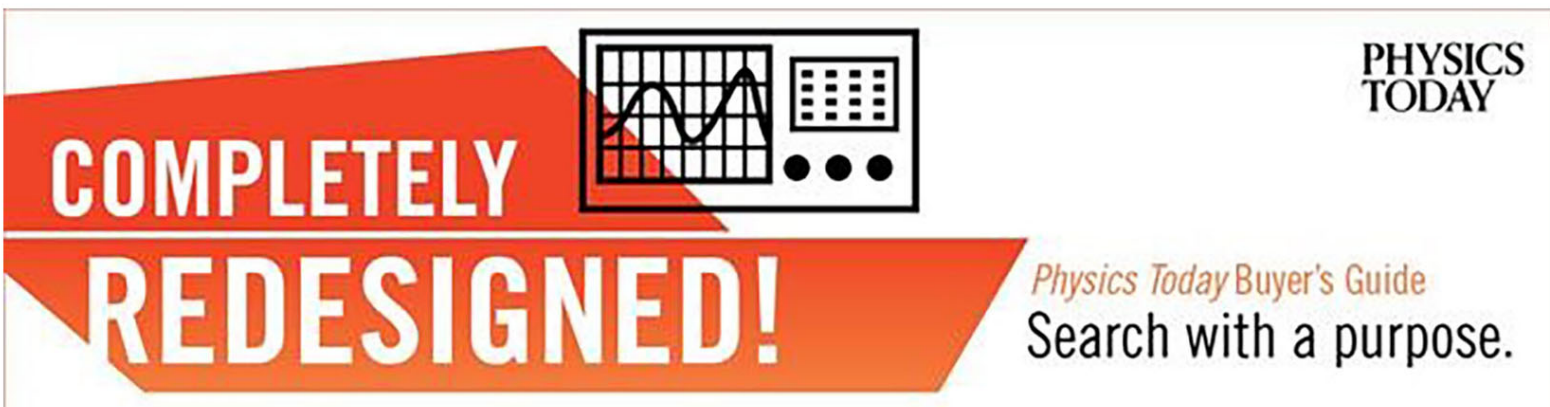




\title{
On the multistream approach of relativistic Weibel instability. III. Comparison with full-kinetic Vlasov simulations
}

\author{
A. Ghizzo \\ Institut Jean Lamour UMR 7163, Université de Lorraine, BP 239 F-54506 Vandoeuvre les Nancy, France
}

(Received 16 May 2013; accepted 15 July 2013; published online 9 August 2013)

\begin{abstract}
The saturation of the Weibel instability in the relativistic regime is investigated within the Hamiltonian reduction technique based on the multistream approach developed in paper I in the linear case and in paper II for the nonlinear saturation. In this work, the study is compared with results obtained by full kinetic 1D2V Vlasov-Maxwell simulations based on a semi-Lagrangian technique. For a temperature anisotropy, qualitatively different regimes are realized depending on the excitation of the longitudinal (plasma) electric field, in contrast with the existing theories of the Weibel instability based on their purely transverse characters. The emphasis here is on gaining a better understanding of the nonlinear aspects of the Weibel instability. The multistream model offers an alternate way to make calculations or numerical experiments more tractable, when only a few moments of the velocity distribution of the plasma are considered. C 2013 AIP Publishing LLC. [http://dx.doi.org/10.1063/1.4817752]
\end{abstract}

\section{INTRODUCTION}

In our Refs. 1 and 2, further referred as papers I and II, we have extended the multistream model to the treatment of the Weibel instability (WI) in the relativistic regime. This Hamiltonian reduction technique is an valuable theoretical tool for analyzing not only the linear regime of WI in Paper I in Ref. 1 in the relativistic regime but also in nonlinear situations when both electric and magnetic trappings play a key role at the saturation as shown in paper II in Ref. 2. WI is an instability that does not rely on selective particle resonances and is characterized by persistent modes having non propagating spatial wave structures that require only a slight anisotropy in the distribution function to exist. Whereas WI is known to be purely electromagnetic and non resonant, a few simple theoretical predictions can be made about the coupling with the electrostatic field when the stream symmetry is broken. Thus, when the plasma distribution function is not only anisotropic but also dissymmetric, then there is a marked change in the features of the instability because now the coupling with the electrostatic branch is possible.

As the WI develops, we expect that the distribution function changes in the $\boldsymbol{p}$ momentum space, so that the degree of anisotropy decreases and the instability exhibits saturation. However, the saturation mechanism remains a fundamental plasma physics open question. The dynamics of saturation of WI has been insufficiently studied until now, especially in the relativistic regime and with respect to the generation of quasi-state coherent (probably unstable) magnetic vortices in phase space. The first theoretical studies of stationary solutions during the saturation regime of WI date back to the 1970's in Ref. 3, when it was recognized that (pure) magnetic Bernstein-Greene-Kruskal (BGK) equilibria can be formed in the non relativistic regime for a circularly polarized magnetic field. This sort of behaviour is highly reminiscent of the nonlinear equilibrium solution of Akhiezer and Polovin ${ }^{4}$ met in relativistic laser-plasma interaction, which strongly indicates that the decoupling between longitudinal (electrostatic) and transverse (electromagnetic) contributions can be found only for circularly polarized electromagnetic wave. Furthermore, in the case of a linear polarization, this type of equilibrium must include an electrostatic contribution and it becomes now difficult to separate the magnetic and electric trapping processes.

It is usually expected that the saturation of WI through magnetic trapping sets in, once the magnetic bounce frequency becomes comparable to the linear growth rate of the instability. Indeed the situation is probably more complex, since we have shown in paper II that each population of particles, located inside a given "stream," can experience its own bounce frequency depending of the local canonical invariant $\boldsymbol{P}_{c \perp}$. Thus, within the quasilinear framework, the concept of bounce frequency of the whole system induced by magnetic trapping must be interpreted as a "mean" value connected to the interaction between streams. Indeed, the streams do not interact with each other directly, but are subject to the same electrostatic field in addition to the Lorentz force. In particular, the electrostatic component is, in turn, induced by the collective space charge of all the streams. Thus, the effects induced by the plasma field cannot be neglected.

Furthermore, a critical role in the complex interaction between magnetic-type BGK structures and plasma is expected to be played by the presence of such large selfgenerated electrostatic fields. Longitudinal plasma fields can have profound influence on the resonant character of particle-wave interactions. An extremely surprising aspect arises on the long-time dynamics of trapped particles, which may trigger secondary electrostatic-type instabilities such that the longitudinal two-stream instability recently observed in Particle-In-Cell (PIC) simulations in Refs. 5 and 6, Vlasov simulations in Refs. 7 and 8 or within the framework of the multistream model in Ref. 9 in the non relativistic regime of WI. However, the existing kinetic codes (both PIC and 
Vlasov) cannot account for effects caused by trapped particle populations of very low densities. In particular, those effects require a very accurate numerical treatment and the codes may not render the underlying physics transparent. Therefore, it would be more beneficial to use a reduction multistream technique, when available, to accommodate trapped-particle effects.

In the Vlasov simulations discussed in II based on a multiring model (for a circular polarization of the electromagnetic field), the main process of saturation begins with magnetic trapping with eventually pair-wise merging of phase space trapping structures leading to a "downward chirp" in wave number. This is the process which is seen to give a stable state characterized by a magnetic version of a BGK equilibrium established on the mode $k_{0}$. At later time, charge effects lead on the growth of the electrostatic counterpart, which is also made on the same mode $k_{0}$ allowing to maintain the stability of the BGK structure. However, if the dominant mode differs from $k_{0}$ (in that case, the plasma does not see the action of the ponderomotive field purely on the mode twice of $k_{0}$ in presence of electrostatic fluctuations), a reorganization of the plasma is observed. Thus, a selfreorganization of the plasma, at saturation, begins with a symmetry-breaking instability of pair-wise merging of phase space vortices, where the plasma adjusts itself in wave number to give a stable state dominated by trappings of both electric and magnetic nature. In the case of a linear polarization, we expect that the mode $2 k_{0}$, induced by the Lorentz force, plays a key role by imposing a strong contribution of the mode $2 k_{0}$ on the electrostatic plasma field component, leading to the destabilization of the magnetic BGK equilibrium. Guided by these first analytical predictions based on our multistream model in paper II, the main purpose of this article is to investigate the nonlinear saturation regime of WI by means of simulations based not only on our reduced Hamiltonian model but also by using full-kinetic $1 \mathrm{D} \frac{1}{2}$ (i.e., 1D2V) Vlasov simulations. Here, the emphasis is put on the regime of strong electric and magnetic coupling, which takes place in the case of a linear polarization. Thus, numerical experiments will be performed in the framework of a semiLagrangian scheme, which allows fine description of the dynamics of trapped particles. The paper is organized as follows. Section II is devoted to the study of WI in the nonrelativistic regime allowing us to recall the main features of the topology of nonlinear solutions met in the whole phase space. The case of the relativistic regime is then studied in Sec. III with a detailed description of the particle dynamics afforded by both models. Finally, Sec. IV is reserved for the conclusions.

\section{PHYSICAL FEATURES OF THE WEIBEL INSTABILITY IN THE NON RELATIVISTIC REGIME}

Before describing the numerical results obtained by our kinetic Vlasov codes, let me recall the main features of our numerical scheme. Because the numerical integration of the Vlasov equation is one of the key challenges of computational plasma physics, an intensive work on this subject has produced many different numerical schemes, which however can be bunched together in two main groups. On the one hand, PIC codes have proven to be useful in studying plasma dynamics even for 2D or 3D problems and complex geometries. However, only a few particles per cell have been used particularly in 3D PIC codes leading to a high level of numerical noise, especially in regions of phase space where the density is low. These PIC codes may largely overestimate the plasma heating because of intrinsic "numerical heating." On the other hand, semi-Lagrangian Vlasov codes display an extremely low level of numerical noise but demand a stronger numerical effort than their particle counterparts and require the discretization of the whole phase space.

In both 1D2V Vlasov plus multistream codes, used here, the numerical algorithm is based on a semi-Lagrangian scheme detailed in Ref. 10 allowing the integration of the Vlasov equation directly in phase space. The semi-Lagrangian Vlasov solver implies, for a time step,

(i) to determine the "particle" motion along the backward characteristics and

(ii) to compute the value of the distribution function at the origin of the characteristics by reconstructing the distribution function using B-spline or cubic-spline interpolation schemes from the different mesh point values.

It must be pointed out that semi-Lagrangian Vlasov simulations ${ }^{13}$ are slowly introduced in place of the well-known Lagrangian PIC simulations for two main reasons: the lack of numerical noise and the very good resolution of the distribution function in phase space provided the dimension of the momentum space is low as possible. The first property makes "Vlasov" codes a powerful tool to study the growth of instabilities of laser-plasma interaction even in 2D problem in Refs. 11 and 12. Second, the very good resolution in phase space allows a precise study of wave-particle interactions including particle trapping and particle acceleration.

We now briefly illustrate the physical mechanism for the thermal anisotropy-driven Weibel instability. Motivated by direct numerical comparisons, the case of a linear polarization of the electromagnetic field is only considered here. Notice that, in that case, the coupling between the transverse electromagnetic and electrostatic branches of the dispersion relation is strong (as shown in paper I). We carry out 1D2V Vlasov simulations of WI, first in the non relativistic regime. A set of numerical simulations of WI has been performed by using, as initial conditions, Maxwellian distribution functions with a temperature anisotropy corresponding to $T_{x}=1 \mathrm{keV}$ and $T_{y}=50 \mathrm{keV}$ along the longitudinal $p_{x}$ and perpendicular $p_{y}$ components, respectively. We first consider a single unstable mode $k_{0}$. The numerical space domain, in dimensionless units, is given by $L=2 \pi / k_{0}$. Here, we choose $k_{0} d_{e}=1.75$. We perturb the system by a magnetic field term $\delta B_{z}=b_{0} \sin k_{0} x$ with $e b_{0} / m \omega_{p}=10^{-4}$ as initial amplitude. The phase space sampling for the full kinetic 1D2V Vlasov simulation (here denoted the V-model) is $N_{x} N_{p_{x}} N_{p_{y}}$ equal to $256^{3}$ and we choose a time step of $\triangle t \omega_{p}=0.005$. Although in general WI can occur within a range of wave number $k$, the fact to introduce here a perturbation on a single mode is not only an excellent opportunity to compare the analytic 
theory with numerical simulations but may also provide an insight of how a more realistic multimode plasma evolves and saturates. It must be pointed out that it is the noiseless character of the semi-Lagrangian scheme that affords such a possibility since other modes remain at the level of round-off errors during the simulation.

From a physical point of view, the picture used in the multistream $(\mathrm{mS})$ model is highly reminiscent of the one due to B. Fried in Ref. 14 of WI in which a magnetic field perturbation is made to grow. For simplicity, we describe the dynamics of the electrons only and assume that ions are fixed providing global charge neutrality. Let us consider two opposite streams $C_{-j}$ and $C_{j}$ among the streams, with equal particle fluxes in opposite directions, i.e., a "symmetrical" case with $C_{-j}=-C_{j}$, so that the net current is zero for both considered streams. The introduction of a small perturbation of the magnetic field leads to the separation of the currents in space, thereby producing the amplification of the magnetic field. Thus, both currents and magnetic fields increase exponentially. Even though it is a problem clearly stated, theoretical and numerical simulations reveal novel and surprising features of the instability. Indeed, it is these alternating currents that drive the instability and lead to the wellknown Y-shape of the distribution function met in the $x-p_{y}$ phase space, already observed by Stockem et al. in PIC simulation in Ref. 15 or by others in Vlasov simulations in Ref. 9.

The net total current $J_{y}$ created depends on how effectively the "partial" currents of the opposite sign can be reduced by transverse deflection of particle "bunches" away from the region of interest. In Fig. 1, snapshots of the electron distribution function in the $x-p_{y}$ phase space is shown on the middle panel at time $t \omega_{p}=67.5$, i.e., in the saturation phase of WI. The figure exhibits a "layered" Y-shape structure. More interesting are the top and bottom panels, which show the corresponding distribution but now in the $x-p_{x}$ phase space. Here, we have selected the particle distribution for two different values of $p_{y}= \pm 2 p_{y, t h}$, i.e., we have plotted the quantities $f\left(x, p_{x}, p_{y}= \pm 2 p_{y, t h}\right)$ where $p_{y, t h}$ is the thermal momentum along $p_{y}$ (i.e., in the nonrelativistic regime such a quantity is defined by $\left.p_{y, t h} / m c=\sqrt{k_{B} T_{y} / m c^{2}}\right)$. Here, the top panel in Fig. 1 corresponds to the distribution located at $p_{y}=2 p_{y, t h}$ while the bottom panel represents the data of $f$ at $p_{y}=-2 p_{y, t h}$. As revealed by global $1 \mathrm{D} 2 \mathrm{~V}$ Vlasov simulations, an isolated "stream" is formed in the region of high momentum. These particle beams are linked to the property of invariance of the canonical momentum in the perpendicular direction, since in the 1D model, the distribution $f$ does not depend explicitly of the $y$ variable. Not only the results of the nonlinear Vlasov simulations show that the nonlinear saturation is governed by strong magnetic trapping as expected but that the concept of "stream" is important in WI.

Moreover, in magnetic trapping theory (Davidson 1972 in Ref. 16), the instability reaches its saturation level when the magnetic bounce frequency $\omega_{b}$ becomes comparable to the linear growth rate $\Gamma$. The magnetic bounce frequency is then defined in the non relativistic regime by

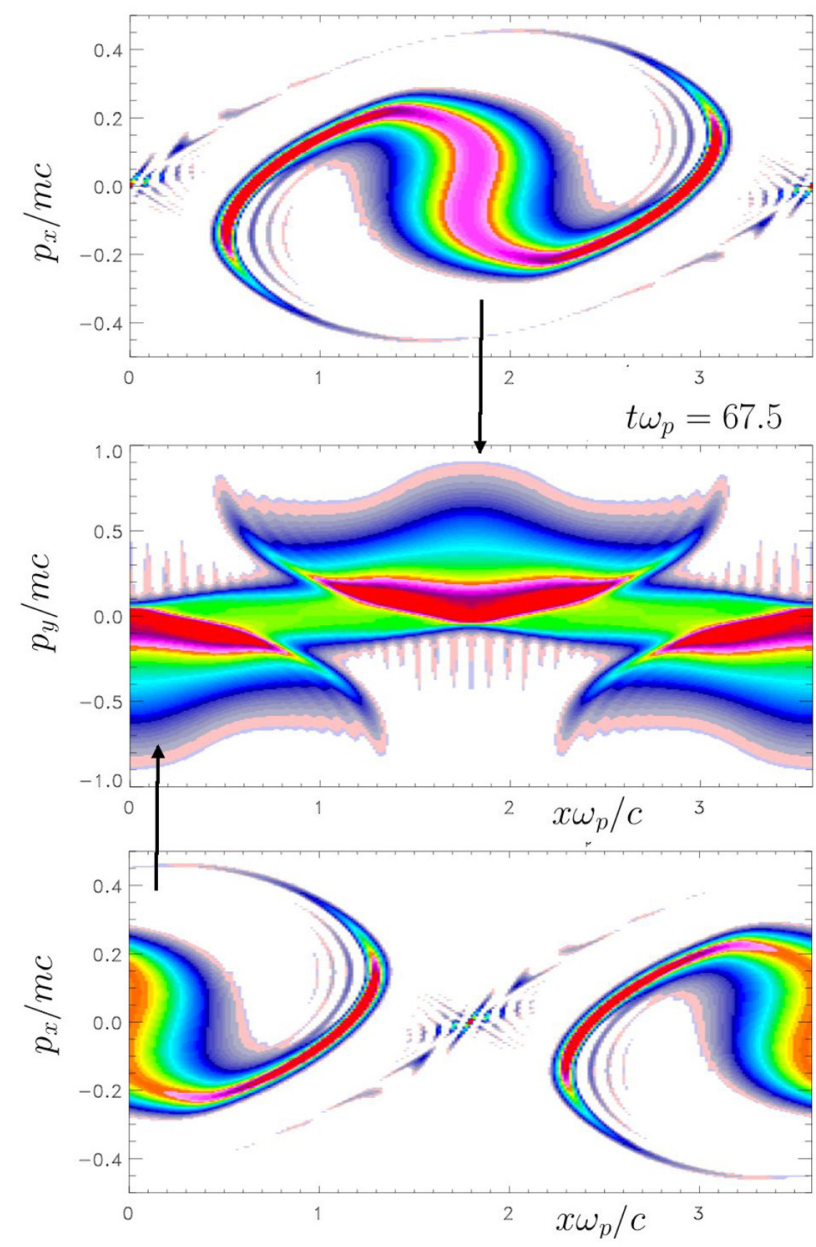

FIG. 1. On middle panel snapshots of the electron distribution function in the $x-p_{y}$ phase space, exhibiting a "layered" Y-shape structure. On top and bottom panels, the selected particle population in the $x-p_{x}$ phase space, corresponding to "streams" located at $p_{y}=+2 p_{y, t h}$ (top) and $p_{y}=-2 p_{y, t h}$ (bottom) where $p_{y, t h}$ is the thermal momentum along $y$. This simulation has been performed using the 1D2V full kinetic Vlasov-Maxwell solver phase space, exhibiting a "layered" Y-shape structure.

$$
\frac{\omega_{b}}{\omega_{p}}=\sqrt{\frac{k_{0} c}{\omega_{p}} \frac{p_{\perp}}{m c} \frac{e B_{\max }}{m \omega_{p}}} .
$$

An estimation of $\omega_{b}$, for a given stream, can be obtained through the data obtained by the Vlasov simulation. Thus, to choose $k_{0} d_{e}=1.75, e B_{\max } / m \omega_{p} \simeq 0.12-$ a typical value of the saturated magnetic field amplitude observed in Vlasov simulation—and to take $p_{\perp} \sim 1.5 p_{y, t h}=0.469 m c$ gives rise to a value of $\omega_{b 1} \simeq 0.31 \omega_{p}$, while the linear growth rate was found close to the numerical value of $\Gamma_{\text {num }}=0.40 \omega_{p}$.

The help of the high resolution phase space diagnostics of the multistream code allows to give a much more precise explanation. For the $\mathrm{mS}$-model, we have used a phase space sampling of $N_{x} N_{p_{x}}$ of $512 \times 1024$ grid points (such a phase space sampling allows a very accurate description of phase mixing). The time step used in the multistream simulation is taken as $\Delta t \omega_{p}=0.0025$. We model the nonlinear evolution of WI by using seven streams in the perpendicular momentum space; each stream being spaced from its neighbouring from $\triangle C=C_{j+1}-C_{j} \sim p_{y, t h}$. Since in the considered case 
here of WI, the macroscopic description requires only the first moments of $f$, only a small number of streams is required here for an accurate description of the instability even in the nonlinear regime. Numerical simulations using the $\mathrm{mS}$-code have been carried out using identical parameter of $k_{0}$ and of $T_{x}=1 \mathrm{keV}$ and $T_{y}=50 \mathrm{keV}$ as previously used in the full kinetic treatment.

In Fig. 2, we show the plot the stream's distribution in phase space at different times during the beginning of the particle trapping process, the stream being initially located at $C_{j}=2 p_{y, t h}$. We see the formation of the rotating trapping vortex in phase space and the occurring of "arms," which are constituted by filaments. We see clearly in Fig. 2 that filaments get thinner ${ }^{17}$ as time goes on due to several rotations of the central trapping structure. Furthermore, the distribution of trapped particle exhibits also a weak modulation on the mode $m=2$ inside the magnetic vortex, as the result of the presence of the Lorentz force on bottom panel.

To explore the physics of the multistream model, we have plotted in Fig. 3 the time evolution of the total magnetic
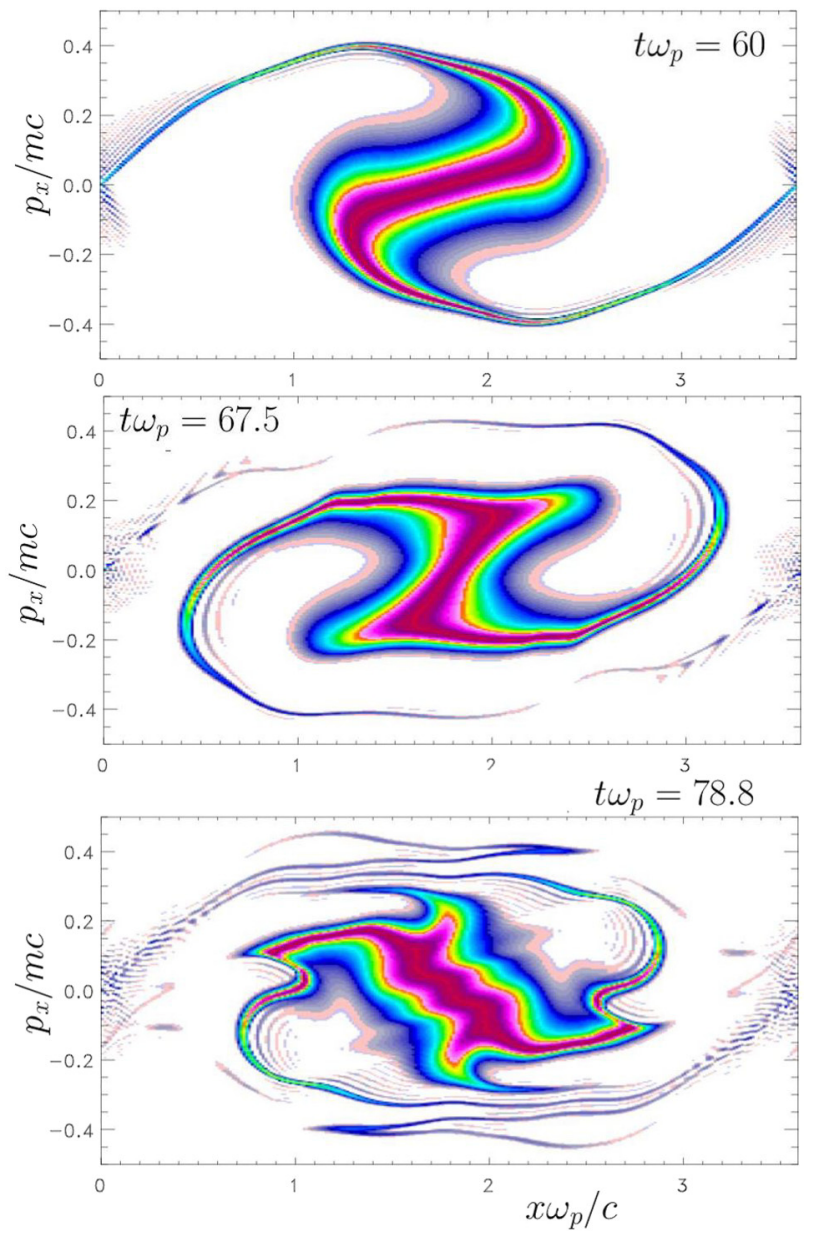

FIG. 2. Phase space behaviour of a selected electron population of the stream initially located at $C_{j}=2 p_{y, t h}$, at different times during the evolution of the system. Results were obtained using the multistream model with seven streams. WI is here driven by a temperature anisotropy of $T_{x}=1 \mathrm{keV}$ and $T_{y}=50 \mathrm{keV}$ in the longitudinal and transverse directions, respectively. Just before the saturation of the instability, particles experience the beginning of a particle trapping mechanism dominated by the magnetic field. We observe here the formation of the magnetic trapping structure rotating in phase space.
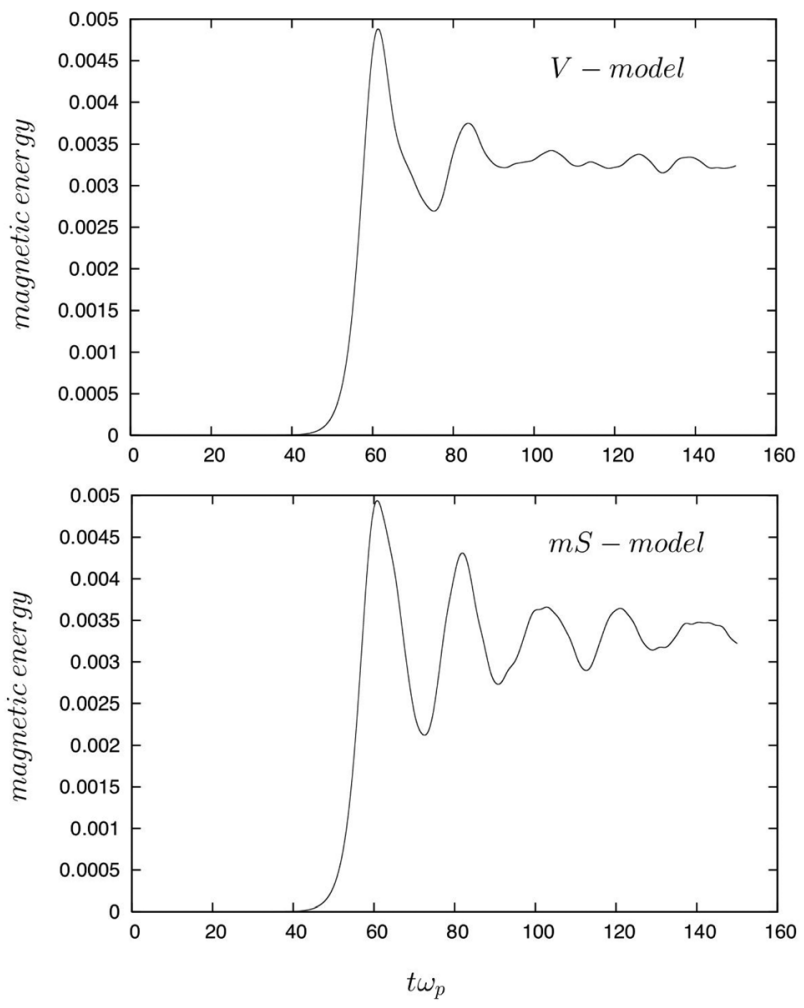

FIG. 3. Time evolution of the magnetic energy obtained, respectively, from the $\mathrm{V}$-model on top panel and the mS-model on bottom panel. The physical parameters are identical in both numerical simulations. The perturbation is made on the magnetic field on the mode $k_{0} d_{e}=1.75$ with a small amplitude of $b_{0}=10^{-4}$ in dimensionless units. The density energy of $B_{z}$ increases in an exponential way in both simulations until time $t \omega_{p}=60$. Thus, after the instability saturates, the amplitude of the magnetic energy density oscillates for a few cycles and the period of oscillation is found to be close to the bounce frequency of $\omega_{b} \simeq 0.308 \omega_{p}$.

energy $\frac{1}{2 \mu_{0} L} \int_{0}^{L} B_{z}^{2} d x$ as a function of time: the version of the full kinetic simulation (the V-model) is shown on the top panel while the result obtained directly by the $\mathrm{mS}$ model is plotted on bottom panel. The density energy of $B_{z}$ increases in an exponential way with the same growth rate of $\Gamma=$ $0.40 \omega_{p}$ in both simulations until $t \omega_{p}=60$. Thus, after the instability saturates, the amplitude of the magnetic energy density oscillates for a few cycles and the period of oscillation is approximately close to $\tau_{b} \omega_{p} \simeq 20.37$, i.e., corresponding to the numerical bounce frequency of $\omega_{b}=2 \pi / \tau_{b}$ $\simeq 0.308 \omega_{p}$, which is also in good agreement with the value determined from Eq. (4), i.e., $\omega_{b 1} \simeq 0.31 \omega_{p}$ for the selected beam. Such an agreement supports the argument that magnetic trapping is responsible for the saturation of a single unstable mode and that the $\mathrm{mS}$ model is capable to recover the saturation mechanism by using a small number of streams. It must be pointed out the oscillation of the magnetic field energy is clearly recovered using the mS model and is accompanied by a somewhat weak damping around an asymptotic saturated value of $\varepsilon_{B} \simeq 0.0034$, identical in both kinds of simulations.

Although the number of streams is restricted in the multistream simulation, compared with the full kinetic one, these streams are however described with a high level of accuracy, allowing to recover the oscillatory damping process observed 
in the time evolution of the magnetic energy in the saturation regime as can be seen on bottom panel in Fig. 3. Such features signify an important role of stream effects on the saturation mechanism of WI. One of the major merits of the $\mathrm{mS}$ model is to get a comprehensive understanding of magnetic field generation and its feedback mechanism on the electrostatic field generation. If one makes the further assumption that the electrostatic activity was linked to asymmetric behaviour of the particle distribution in the perpendicular momentum direction, then an accurate description of high velocity streams might be required for the fine description of the amplification mechanism of the electrostatic energy. In the nonlinear phase, each stream is subject to the same wave-breaking scenario (connected to the magnetic trapping process) but experiences its own magnetic bounce time scale since Eq. (4) indicates that the bounce magnetic frequency depends on the local value of the canonical momentum $P_{C \perp}$. Thus, each stream $j$ "sees" a different bounce frequency $\omega_{b j}$ depending on the initial invariant $C_{j}$. This fact induces a symmetry-breaking on $p_{y}$ in the nonlinear regime between a couple of chosen invariants $\left\{C_{j}, C_{-j}\right\}$.

In contrast to the full-kinetic model, the number of "streams" can be considered as infinite, so that an accurate description of high velocity streams becomes difficult due to the numerical dissipation and because the particle "bunches" are located in the small densities regions of the distribution function. Thus, in the V-model, the derivative operator $\partial / \partial p_{y}$ has to be approximated by a dissipative numerical scheme and we are faced with the usual sampling problem. Due to their inherent numerical noise, the problem is probably worse in PIC codes. On the other hand, a multistream description can be used even a small number of streams with a significant reduction of the numerical effort.

\section{STUDY OF WI IN THE RELATIVISTIC REGIME}

\section{A. 1D2V semi-Lagrangian Vlasov simulations in the relativistic regime of $\mathrm{WI}$}

Numerical simulations of WI have been performed in the relativistic regime, using the $1 \mathrm{D} 2 \mathrm{~V}$ full kinetic simulations, with an initial equilibrium distribution function of Schlickeiser given in Refs. 18 and 19 defined by

$$
F\left(u_{x}, u_{y}\right)=C e^{-\mu E-\alpha u_{x}^{2}}=C e^{-\mu\left(E+\psi u_{x}^{2}\right)},
$$

where the parameter $\mu=m c^{2} / T_{y}$ characterises, in the standard way, the perpendicular plasma temperature and $\alpha=\mu \psi$ introduces the temperature anisotropy of the distribution. Here, the new variable $E=\sqrt{1+u_{x}^{2}+u_{y}^{2}}$ is the normalized energy to $m c^{2}$ and $u_{x / y}=p_{x / y} / m c$ is the normalized momentum. Note that for $\alpha=0$, the distribution (2) reduces to the isotropic Maxwell-Jüttner distribution. $C$ is a normalization constant (see Ref. 18 for more details) determined by imposing the condition $\iint d^{2} u F\left(u_{x}, u_{y}\right)=1$. The perpendicular electron temperature is chosen to $T_{y}=2000 \mathrm{keV}$ and WI is here driven by a temperature anisotropy defined by the parameter $\psi=4$. The distribution function is perturbed by a magnetic fluctuations on the $B_{z}$ component of the magnetic field by using

$$
\delta B_{z}=\sum_{m=1}^{M} b_{0} \sin \left(\frac{2 \pi m x}{L}+\varphi_{m}\right),
$$

where $\varphi_{m}$ are random phases and $e b_{0} / m \omega_{p}=10^{-5}$ is the normalized mode amplitude. We have taken $M=20$ modes and a plasma length of $L=16 \pi d_{e}$. The phase space sampling used here is $N_{x} N_{p_{x}} N_{p_{y}}=1024 \times 193^{2}$ while the time step is chosen to $\triangle t \omega_{p}=0.025$. The boundaries on the momentum components on $p_{x}$ and $p_{y}$ are, respectively, given by $\pm p_{x, \max }= \pm 7.5 \mathrm{mc}$ and $\pm p_{y, \max }= \pm 15 \mathrm{mc}$, respectively.

Fig. 4 shows the time evolution of the magnetic energy $\frac{1}{2 \mu_{0} L} \int_{0}^{L} B_{z}^{2} d x$ on top panel and the corresponding electrostatic energy $\frac{1}{2 L} \int_{0}^{L} \varepsilon_{0} E_{x}^{2} d x$ in linear scale versus time on bottom panel. We see that the magnetic energy density of $B_{z}$ increases exponentially in time until $t \omega_{p} \simeq 50$. After the initial exponential growth, the magnetic energy continues to grow in time and with a low frequency modulation until time $t \omega_{p}=100$ and then saturates for $t \omega_{p} \geq 100$. It must be pointed out that the growth of the magnetic energy is accompanied by a strong increase of electrostatic activity. On the other hand, the energy density of both inductive and electrostatic field components rapidly decrease in time after the
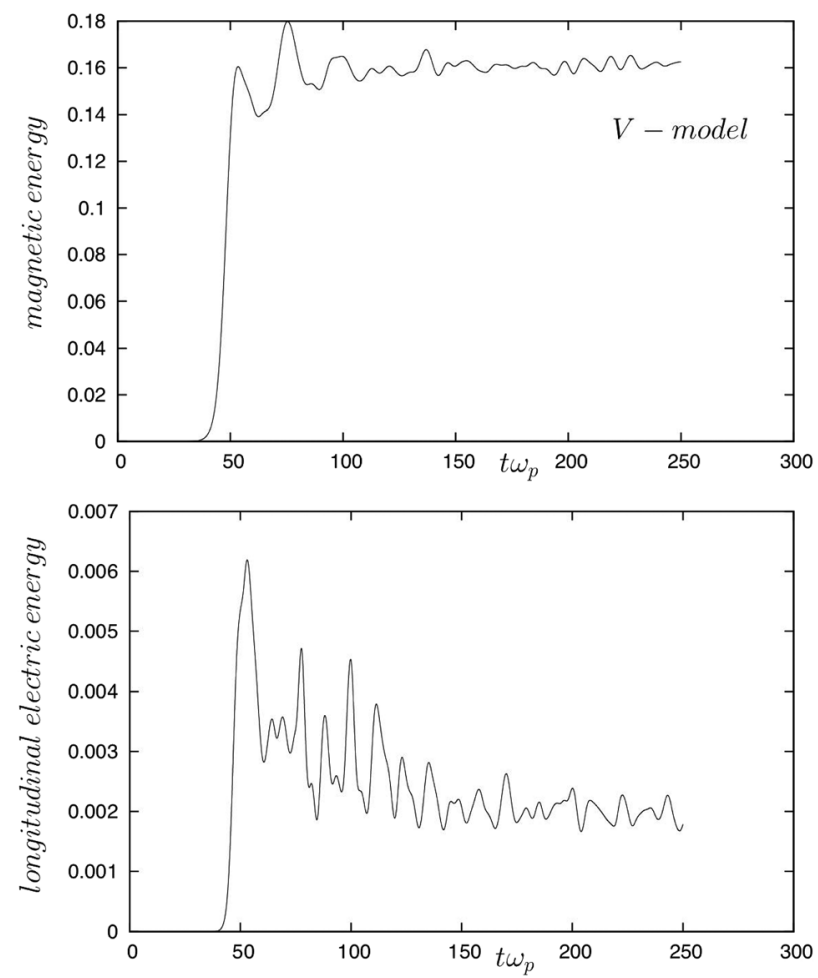

FIG. 4. Temporal evolution of the magnetic energy (top panel) and of the electrostatic part (bottom panel). The physical parameters are $T_{y}=2000 \mathrm{keV}$ with a temperature anisotropy of $\psi=4$ using the Schlickeiser's distribution as indicated in Ref. 18. The growth of the magnetic energy is accompanied by a strong increase of electrostatic activity. Furthermore, the energy density of the electrostatic energy rapidly decreases after saturation. Here, the estimated growth rate of this initial phase was found to be close to $\Gamma / \omega_{p} \simeq 0.22$. Results were obtained using the $\mathrm{V}$-model with full kinetic effects. 
initial step of exponential growth observed for all magnetic plus electric components. Here, the estimated growth rate of this initial phase was found to be close to $\Gamma / \omega_{p} \simeq 0.22$.

We anticipate Sec. III B and quote the simplest results. There are actually two effects. The first effect is connected again to the magnetic trapping process. However, in a purely transverse Weibel scenario, there is no wave-particle resonance since the real part of the frequency is zero. As we will see later, the instability is here a mixture of transverse and longitudinal electric modes, leading to the growth of the plasma electric energy. The second effect is a selfreorganization of the magnetic field $B_{z}$ in terms of an inverse-type cascade process. In many aspects, the results presented here are similar to those observed for WI using PIC simulations in the non relativistic regime in Ref. 5 or in full relativistic regime in Refs. 6 and 20. For instance, the shift of the spectrum to lower wave number $k$ and the saturation into a quasi-steady state have been already observed in Ref. 20. However in Ref. 6, it is worth mentioning that a naive extension of the bounce frequency to the relativistic case would lead to a substantial discrepancy between theory and PIC simulations. The plasma evolution, labelled here "inverse cascade" process usually refers to a continuous flow towards lower wave numbers although perhaps, "downward chirp" might be more appropriate since the chirp is seen for the transition in wave number from $m=7$ till $m=5$. Furthermore, the saturation regime is associated with a strong heating in the longitudinal direction. In the initial stage of the instability, the kinetic energy is transferred to the magnetic field. After saturation, part of the energy stored in the electromagnetic field is transferred back to the plasma particles, which leads to strong heating in the longitudinal direction (here the $p_{x}$ component). The distribution function with an initial temperature anisotropy is unstable to WI whose consequence is to reduce the anisotropy.

The one important question remaining is the role played by the electrostatic fluctuations required to understand the saturation of WI. It is not yet clear whether the observed quasi steady magnetic BGK state predicted at the saturation are stable. It was also observed in Ref. 20 in PIC simulations that particles in the high energy tail of the distribution isotropize at much slower rates that particle population taken in the bulk of the distribution. Although they are minority in number, these high energy particles (which indeed constitute "high velocity streams") can have a significant contribution. At the moment, the effects induced by the resonant waveparticle interaction are still an open question. Another aspect met at the saturation is the origin of the growing electrostatic field. In Ref. 15, the authors show that this electrostatic component is driven by the magnetic pressure gradient, which can redistribute particles in space.

\section{B. Detailed phase space representation afforded by the V-model}

In the multistream model as argued in paper II, it is thus possible to divide the particle population into classes or particle "bunches" according to their initial perpendicular canonical invariant $C_{j}$ and to follow the evolution of each particle class. Such numerical simulations based on the use of the multistream model will be shown later in the Subsections IIIC and IIID. Such an approach not only allows to recover the well-known result of Davidson et al. in Ref. 16 with regards to the magnetic trapping scenario at saturation but also to go beyond and understand the nature of the particle "heating" in the longitudinal direction. In paper II in Ref. 2, an interesting result is given using the mS-model showing that each particle bunch experiences its own bounce frequency in the magnetic trapping scenario, which in the relativistic regime and for deeply trapped particles, writes as

$$
\frac{\omega_{b j}}{\omega_{p}}=\sqrt{\frac{k_{0} c}{\omega_{p}} \frac{C_{j}}{m c \gamma_{0 j}^{2}} \frac{e B_{\max }}{m \omega_{p}},}
$$

where $\gamma_{0 j}$ is the Lorentz factor approximated by $\gamma_{0 j}=\sqrt{1+\frac{C_{j}^{2}}{m^{2} c^{2}}}$.

Moreover, the $1 D 2 V$ distribution $f\left(x, p_{x}, p_{y}\right)$ may be represented graphically by selecting different values of the momentum component $p_{y}$, which can be connected to the property of invariance of the canonical momentum $p_{y}+e A_{y}$ $=$ const. Notice that the constant can be being chosen to be equal to $C_{j}$ for a given stream noted $j$, making possible a direct comparison with the multistream simulation when $A_{y}$ is negligible. Figs. 5 and 6 display the behaviour, at three different times, of two selected particle streams initially chosen first for a perpendicular momentum $p_{y}$ equal to half the thermal momentum $0.5 p_{y, t h}$ and second for $1.5 p_{y, t h}$. Here, $p_{y, t h}$ denotes the thermal momentum defined in paper $\mathrm{I}$ as $\mu^{-1}=\frac{T_{y}}{m c^{2}}=\sum_{j=-N}^{+N} \frac{\alpha_{j} C_{j}^{2}}{m^{2} c^{2} \Gamma_{j}}$, which becomes here (with $2 \alpha=1) \frac{2 p_{y, t h}^{2}}{m^{2} c^{2} \gamma_{t h}}$, leading to a value of $p_{y, t h} \simeq 4.032 m c$ for a temperature of $T_{y}=2000 \mathrm{keV}$. The "stream" picture is particularly clear in the $x-p_{x}$ phase space.

Fig. 5 shows the time evolution of the electron distribution function corresponding to the former stream, i.e., the one located at $p_{y}=\frac{1}{2} p_{y, t h} \simeq 2 m c$, obtained by the data of the electron distribution function using the V-model. The phase space structure reveals the formation of trapped particle structures induced by the magnetic field. The available simulation results suggest that each stream experiences a bounce trapping frequency depending of the parameter $C_{j}$. It seems that the bounce frequency proves to be well approximated by Eq. (4). Choosing $k_{0}=5 \triangle k=0.625 \omega_{p} / c$ and a magnetic field amplitude of $e B_{z \max } / m \omega_{p} \simeq 0.80$ (a value typically observed in our simulation) yields to a frequency of $\omega_{b 1}=0.448 \omega_{p}$. Thus, the normalized trapping period $\tau_{b} \omega_{p}$ is close to 14.02 indicating that the final time of the simulation corresponds in fact at 18 periods. The initial configuration evolves slowly but at time $t \omega_{p}=50$, the formation of the trapping structure starts. At time $t \omega_{p}=62.5$, one sees the beginning of the coalescence process of the two first magnetic structures at left on top panel in Fig. 5. Notice that a second pair-wise vortex merging takes place in the central region of the system. The fusion of vortices is achieved at time $t \omega_{p}=100$ and the system exhibits then five magnetic structures, which remain till the end of the simulation, strengthening the conjecture of a stationary solution in the 

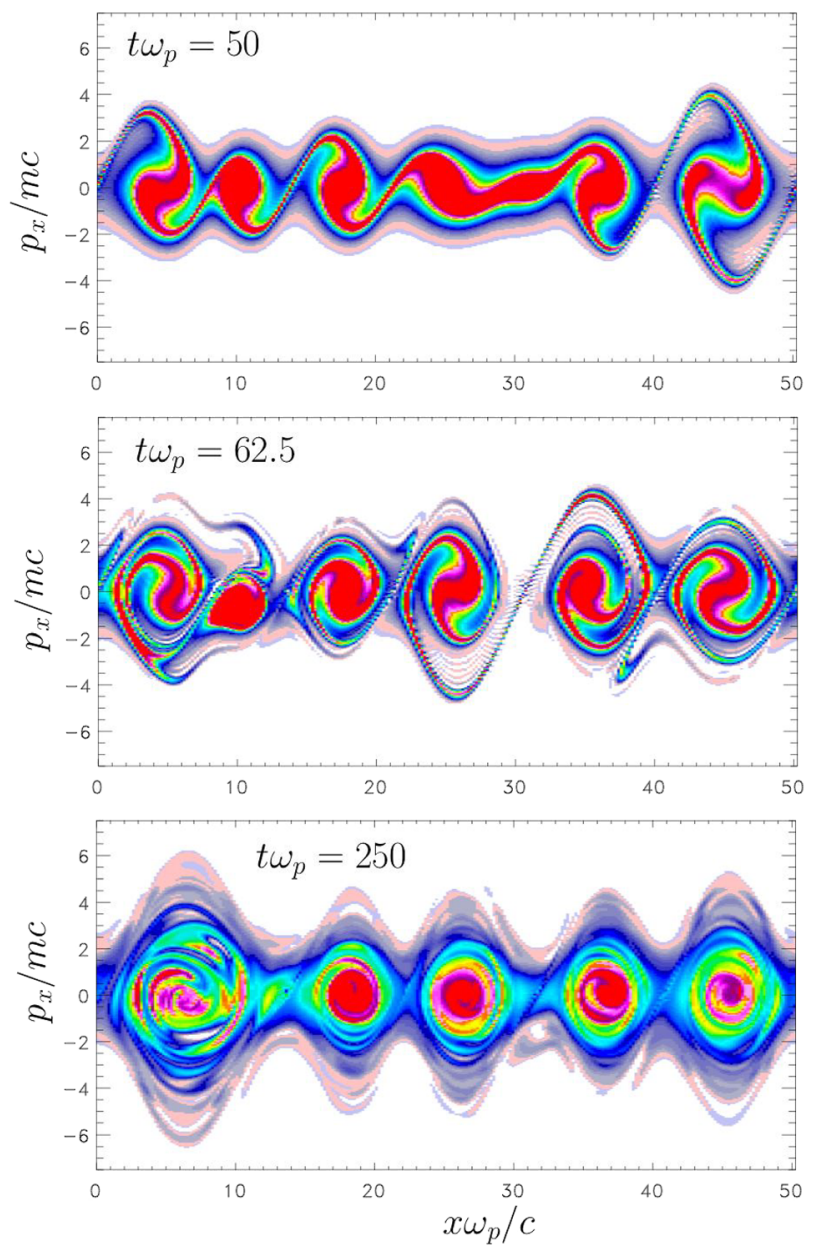

FIG. 5. Phase space representation of the distribution function in the $x-p_{x}$ space for a given value of the $p_{y}$ momentum component chosen at one half of momentum $p_{y, t h} \simeq 2 \mathrm{mc}$. The initial configuration evolves slowly but at time $t \omega_{p}=50$, the formation of the trapping structure starts. On middle panel, one sees the beginning of the coalescence process of the two first magnetic structures at left. When the fusion of the two vortices is achieved, the system exhibits a mode $m=5$ on the bottom panel. Results were obtained using the V-model using a perpendicular temperature of $T_{y}=$ $2000 \mathrm{keV}$ and a temperature anisotropy of $\psi=4$ using the Schlickeiser's distribution, corresponding to Fig. 4.

asymptotic limit. An interesting result is the rather smooth magnetic structures in the final state in phase space at time $t \omega_{p}=250$ after 18 bounce temporal periods.

Similar results can be obtained now for the latter considered "stream," initially chosen at a value of $p_{y}=\frac{3}{2} p_{y, t h}$, which is now plotted in Fig. 6 at the same times. Again the inverse-type cascade is observed till the formation of the five magnetic vortices shown, at the final time, on bottom panel in Fig. 6. In contrast to the previous case shown in Fig. 5, it is observed in Fig. 6 that the rate of "cleaning" of magnetic vortices seems to decrease with increasing perpendicular energy of the stream. In other words for the low energy population, the "cleaning" is faster than the one observed in the case of the high energy stream. In fact, the phase mixing remains identical but the number of phase space rotations differs, thus the former stream, of low energy, reaches a steady state more rapidly. This is due to the fact that, in the case of the high energy particle population, trapped particles experience a lower bounce trapping frequency. For instance
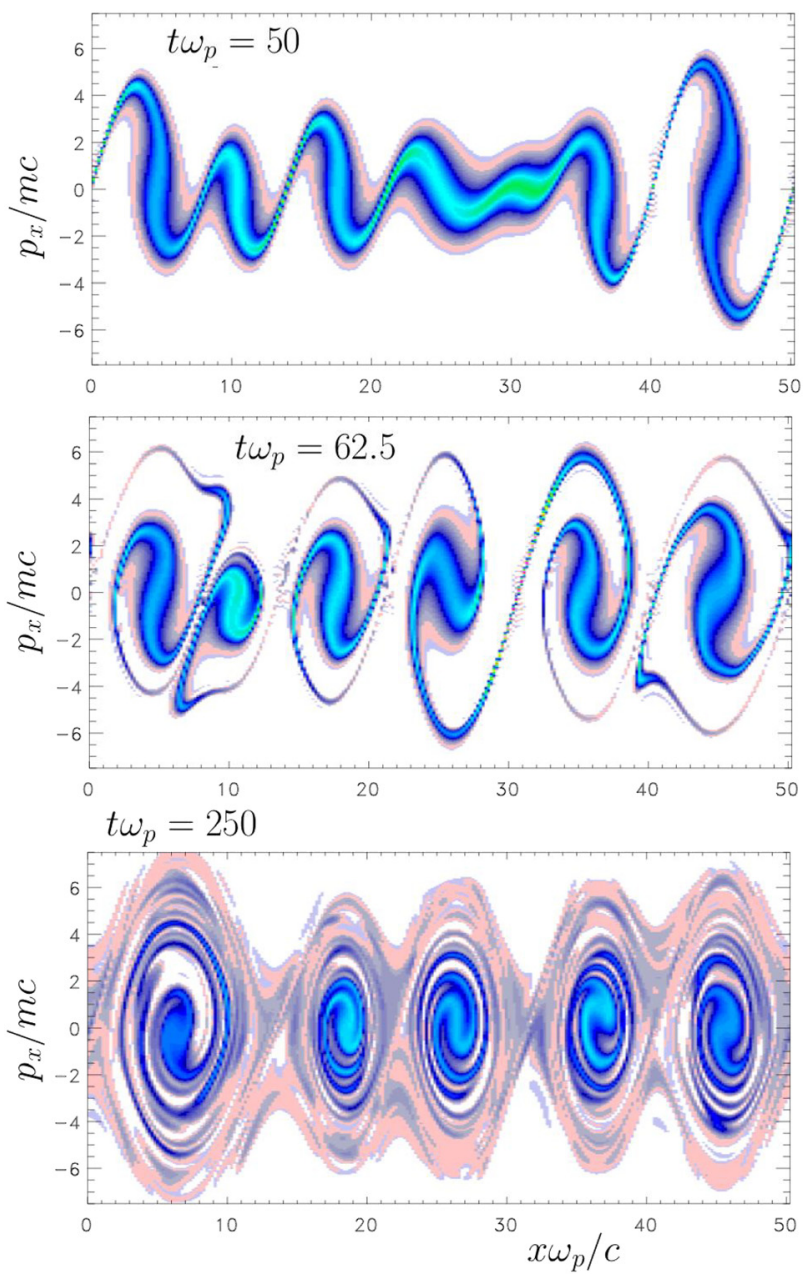

FIG. 6. Phase space representation of the distribution function in the $x-p_{x}$ space for the second selected stream now chosen at $p_{y}=\frac{3}{2} p_{y, t h} \simeq 6 m c$. The observed behaviour corresponds to the combined action of the bounce frequency variation induced by stream velocity, compensated for the reorganization of the plasma in the form of an inverse-type cascade scenario. The physical parameters are $T_{y}=2000 \mathrm{keV}$ with a temperature anisotropy of $\psi=4$ using the Schlickeiser's distribution. Results were obtained using the V-model.

keeping $k_{0} c / \omega_{p}=0.625$ (i.e., the wave number value of the mode $m=5$ ) and a value of the maximum magnetic field amplitude of $e B_{\text {zmax }} / m \omega_{p}=0.80$, the bounce trapping frequency is then close to $\omega_{b 2} \simeq 0.286 \omega_{p}$ for $p_{y}=\frac{3}{2} p_{y, t h}$ $\simeq 6 m c$. The corresponding bounce period is then $\tau_{b}=11.36 \omega_{p}^{-1}$, and the system has known only 12 periods at the same time $t \omega_{p}=250$.

The structures resulting from WI growth are characterized by the formation of strong trapping vortices in the stream distribution. Such vortices have in effect modified the plasma properties enough so that an electrostatic activity starts to grow eventually leading to the coupling with Langmuir waves. In the Vlasov simulations, discussed here, the main process linked to the plasma reorganization begins with the pair-wise merging of magnetic vortices and was here labelled an "inverse-type cascade," although perhaps, "downward wave number chirp" might be more appropriate. Such an instability is usually referred as the electrostatic two-stream instability as in Ref. 5, which begins with a symmetry-breaking instability of pair-wise merging of holes, 
an instability first observed in one-dimensional VlasovPoisson simulations of strong two-beam instability by Bertrand et al. in Ref. 21.

\section{Detailed analysis of WI using the multistream model}

To further investigate the main role played by streams in the saturation of WI, we focus now on numerical experiment afforded by the multistream model. As mentioned previously, the part II of companion paper presents an analysis of the saturation mechanism in terms of mixed electrostaticmagnetic relativistic-type BGK structures. In the $\mathrm{mS}$ model, the description of $p_{y}$ is based on an exact class of solutions. With the aim of obtaining the finest details of the momentum distribution function, rather than using the commonly used PIC model for the Vlasov equation with its inevitable numerical noise, we keep here again the semi-Lagrangian numerical approach as previously used. In the $1 \mathrm{D} 2 \mathrm{~V}$ (V-model) although noise and precision are not severe limits, the momentum resolution in $p_{y}$ requirement for an accurate description of streams of weak densities (and therefore of high $p_{y}$ ) can impose a severe computation burden. The large burden for small density regions is chiefly due to the fact that the fine momentum resolution, although really required for describing many streams, is actually imposed over all momentum space, and even in the full phase space due to relativistic coupling in space and velocity spaces. Thus, due to the invariants $C_{j}$ and the noiseless character of the semiLagrangian approach, our $\mathrm{mS}$ model can give important new insights on the understanding of kinetic processes leading to the saturation or driving the quasi-steady state on long time. By selecting appropriate initial particle "bunches," depending on the problem of interest, the phase space trapping structures can be isolated and examining in detail, allowing to separate the different physical processes characterizing each stream.

After determining that the key mechanism in WI saturation is connected to the dynamics of streams, it is a natural question as to whether the feature of WI can be recovered by using a small number of streams. The results of our simulations show that only seven streams are necessary to reproduce the main physical processes as magnetic trapping, pair-wise vortex merging or amplification of the electrostatic energy. A set of numerical simulations of WI has been performed by using the $\mathrm{mS}$ code for an initial condition based on a temperature anisotropy distribution of $T_{y}=2000 \mathrm{keV}$ and with a parameter $\psi=4$, i.e., a similar initial condition as that previously used in full kinetic Vlasov simulations. We perturb again the system by using a similar magnetic perturbation given in Eq. (3). The other physical parameters are identical to those used in the V-model. The phase space sampling for the $\mathrm{mS}$ model is $N_{x} N_{p_{x}}=513^{2}$. The different streams have been chosen with an identical spacing in momentum of $\Delta C=\frac{1}{2} p_{y, t h}$. Remember that our theory gives good results in the linear analysis in that case (see paper I).

The $x-p_{x}$ phase space dynamics of the chosen stream at $C_{3}=\frac{3}{2} p_{y, t h}$ is shown in Fig. 7 at the same instants of those used in the V-model shown in Fig. 6. The figure shows that
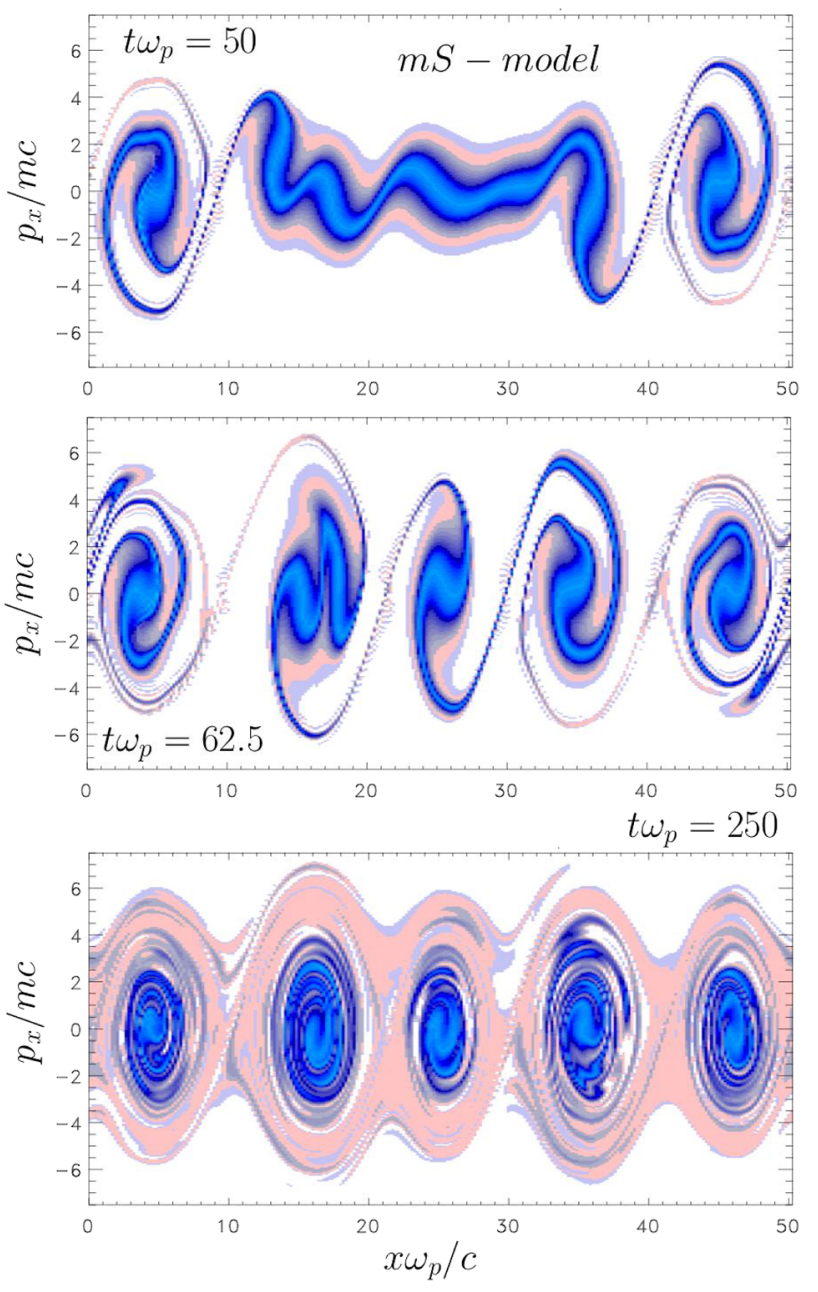

FIG. 7. The $x-p_{x}$ phase space dynamics of the latter stream defined by $C_{3}=\frac{3}{2} p_{y, t h}$. The curve corresponds for plots shown in Fig. 6 obtained with the V-model. Results were obtained using the $\mathrm{mS}$-model in the relativistic regime of WI.

the dynamics is correctly described by the mS-model where only seven symmetrical streams were considered. Again the evolution of the selected particle streams is driven by the self-reorganization of the magnetic field component $B_{z}$ in terms of a downward chirp up to the final mode $m=5$. This process is outlined on top and middle panels in Fig. 7: we observe clearly the pair-wise vortex merging, although the location of the fusion sites differ, now located in region of $10 \leq x \omega_{p} / c \leq 20$ and $30 \leq x \omega_{p} / c \leq 40$. The figures exhibit the transition from the initial state $m=7$ (at time $t \omega_{p}=50$ on top panel), ending to the mode $m=5$ on bottom panel. Thus, even with a small number of streams, our mS-model confirms the existence of the downward chirp in wave number for the magnetic field. The results obtained with the $\mathrm{mS}$ model are very similar with those obtained with the full 1D2V Vlasov model. Notice that there are little modifications on the plasma behaviour due to the initial choice of the random phases introduced in the perturbation term for the magnetic field. Both codes exhibit after the "cleaning" the same final state characterized by five magnetic trapping structures. Simulations show that the plasma reaches a steady state, which appears to coexist self-consistently with the 
electromagnetic field in a stationary magnetic version of the BGK equilibrium.

\section{Dynamics of the central stream}

To further investigate the role of the weak electrostatic field in the reorganization of the plasma as argued in paper II, we now focus on the dynamics of the central stream. Especially, interesting is the double strong modulation of the electron $x-p_{x}$ phase space of the central stream plotted in Fig. 8. Thus, a substantial fraction of the magnetic energy is also converted in the longitudinal electric field. As evident from Fig. 8, particles of the central stream become strongly structured around time $t \omega_{p}=50$ (at that time, the magnetic field energy begins to saturate), indicating that particles are now being trapped by the combined action of the electric potential and the magnetic field. Whereas the electron $x-p_{x}$ phase space develops an oscillatory modulation on the mode $m=5$, the dominant mode is here the mode $m=10$ and therefore ten vortices are formed in phase space. Indeed, we
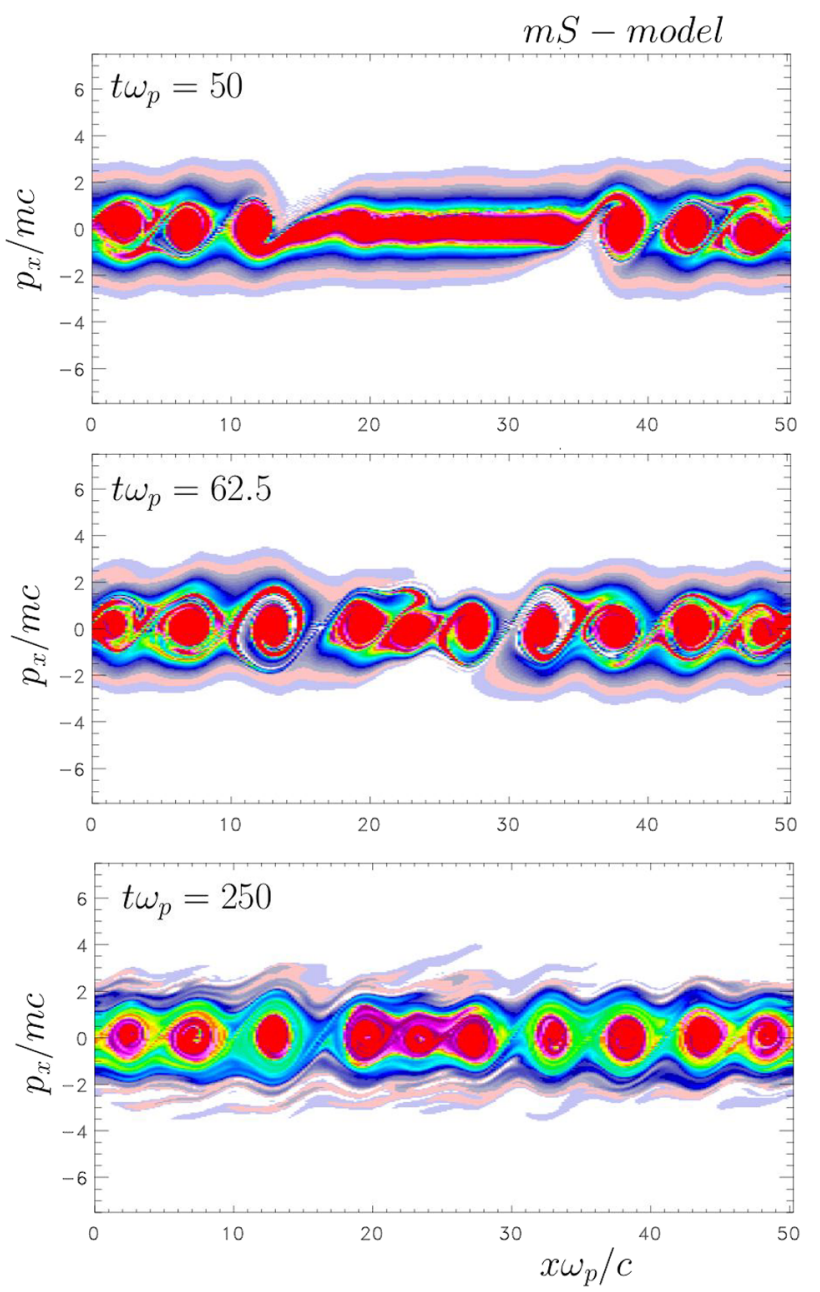

FIG. 8. The $x-p_{x}$ phase space dynamics of the central stream. As evident from a comparison between Figs. 5-7, particles of the central stream exhibits ten trapping vortices at time $t \omega_{p}=250$, with a weak modulation on the magnetic mode $m=5$, indicating that particles are now being trapped by the combined action of the electric potential and the magnetic fields. Ten vortices are clearly visible in phase space indicating that the dominant mode is here $m=10$, i.e., $2 k_{0}$. Results are obtained using the $\mathrm{mS}$-model. do not observe here a vortex merging in the behaviour of the central beam. Since particles of the central beam experience a quasi-zero magnetic trapping $\left(C_{0}=0\right)$, we expect that the dominant mode $m=10$ is driven by the Lorentz force leading to the excitation of the electric field $E_{x}$. Although the electric field component $E_{x}$ is much weaker in intensity when compared to the magnetic field component $B_{z}$, its presence might nevertheless be important, since it could mediate resonant wave-particle interactions. As Fig. 8 shows, despite its low intensity, the growth of $E_{x}$ gives rise to strong particle trapping mechanism, but of electrostatic nature, different of the magnetic trapping mechanism dominant for the other streams. The electrostatic trapping vortices exhibit a double modulation on both the magnetic mode $k_{0}=5 \triangle k$ (i.e., the mode $m=5$ ) but also on twice wave number, i.e., $2 k_{0}$, initially driven by the Lorentz force. Particles reach a dynamical equilibrium in which the Langmuir wave and the magnetic field growth are halted and a stationary state is established. We have also estimated the electrostatic bounce frequency $\omega_{b e}$, which is defined in a standard way by

$$
\frac{\omega_{b e}}{\omega_{p}}=\sqrt{\frac{2 k_{0} c}{\omega_{p}} \frac{e E_{x, \max }}{m \gamma_{t h} \omega_{p} c}} .
$$

We choose $2 k_{0}=10 \triangle k \simeq 1.250$ and $\gamma_{t h}=\sqrt{1+\frac{p_{y, t h}^{2}}{m^{2} c^{2}}} \simeq 4.15$ for a thermal momentum value of $p_{y, t h} \simeq 4.032 \mathrm{mc}$, Eq. (5) gives then a value of the electrostatic bounce frequency of $\omega_{b e} / \omega_{p} \simeq 0.17$. For the estimation of $\omega_{b e}$, we have used here a maximum value of the electric field of $e E_{x} / m \omega_{p} c$ of 0.10 , seen in the multistream simulation.

An interesting point about the electrostatic bounce frequency defined by Eq. (5) is that it is comparable (although somewhat weaker) to the value of the "quasilinear" bounce frequency determined by the magnetic trapping. We have shown in paper II that within the $\mathrm{mS}$-model and for a circularly polarization of the magnetic field, the motion of trapped particles can then be treated as that of a particle in a potential given by

$$
\psi_{j}(x)=\frac{2 C_{\perp j} a_{\perp}}{m c \gamma_{0 j}} \cos \left(k_{0} x-\varphi_{j}\right),
$$

where the canonical invariant is now a vector of type $\boldsymbol{C}_{j}=C_{\perp j} \cos \varphi_{j} \boldsymbol{e}_{y}+C_{\perp j} \sin \varphi_{j} \boldsymbol{e}_{z}$. Here, $a_{\perp}=e A_{\perp} / m c$ is constant in space and $\varphi_{j}$ describes a random phase. The typical time scale of a given stream $j$, characterized by the value of its canonical invariant $C_{\perp j}$, is the bouncing period $\tau_{b j}=2 \pi / \omega_{b j}$, where the bounce magnetic frequency is given by

$$
\omega_{b j}=\frac{\pi}{2 K(\kappa)} \sqrt{\frac{C_{\perp j} a_{\perp} k_{0}^{2}}{m c \gamma_{0 j}^{2}}} .
$$

In (7), the parameter $\kappa$ plays the role of a pitch angle, i.e., for a deeply trapped particle population, we have indeed $\kappa \rightarrow 0$ and $K(\kappa)$ is the complete elliptic function of first kind. We obtain then $K(0)=\frac{\pi}{2}$; and for $\omega_{b j}$, we recover the standard expression given by Eq. (4). 
Coming back to the case of a linearly polarized wave, it is worth mentioning that results from the $\mathrm{mS}$-model analysis indicate, that the averaged bounce frequency due to the magnetic trapping can be obtained in a statistical way by the following expression:

$$
\frac{\bar{\omega}_{b}}{\omega_{p}}=\sqrt{\frac{k_{0} c}{\omega_{p}} \sum_{j=1}^{N} \frac{\alpha_{j} C_{j}}{\left(1+C_{j}^{2}\right)} \frac{e B_{z, \max }}{m \omega_{p}}},
$$

which is close to the value $\bar{\omega}_{b} \simeq 0.239 \omega_{p}$ for $N=3$ (i.e., seven streams). In Eq. (8), $C_{j}$ is expressed in $m c$-units. The expected exponential growth of the excited mode yields to a numerical growth rate close to $\Gamma_{\text {num }} \simeq 0.233 \omega_{p}$ in well agreement with the "quasilinear" value of $\bar{\omega}_{b}$ induced by magnetic trapping. When $\bar{\omega}_{b}$ is comparable to the linear growth rate $\Gamma_{t h}$, the particle trapping becomes a significant effect, in good agreement with the predictions of the quasilinear theory. While our multistream model allows the obtaining of the dispersion relation in linear analysis, this model is also an accurate tool for studying the instability through numerical simulations in the nonlinear regime. The multistream simulations demonstrate that the interaction with the electrostatic component of the electric field can give rise to a self reorganization in the time interval $\left[40,60 \omega_{p}^{-1}\right]$ where both magnetic and electrostatic trapping mechanisms are growing leading to a stable state.

\section{SUMMARY AND CONCLUSION}

This work completes a series of papers describing the physics of the Weibel instability in the relativistic regime. The geometry is here one-dimensional in space coordinate taking advantage from the invariance property of the generalised canonical momentum, i.e., by noticing that, for a given $j$ population, the quantity $\boldsymbol{P}_{c \perp}=\boldsymbol{p}_{\perp}+e \boldsymbol{A}_{\perp}=\boldsymbol{C}_{j}$ is constant in time when the space variable $\boldsymbol{q}_{\perp}$ is lacking. The work is focused on the numerical approach allowed by the model and in particular with numerical comparisons with the full 1D2V semi-Lagrangian Vlasov-Maxwell solver in the relativistic regime of WI.

The multistream model appears to be an interesting alternative to the usual Vlasov kinetic description of the Weibel instability, driven by a temperature anisotropy. Interesting results have been obtained pointing to the ability of the multistream model to depict and resolve kinetic effects in the nonlinear regime of saturation, with the possibility of coupling with the Langmuir wave. The findings of this series of papers should be of general interest to researchers of both inertial fusion and astrophysical communities where strong relativistic effects are expected. To conclude, the multistream approach offers an exact description of the plasma dynamics even with a small number of "streams," providing a relatively simpler analytical framework in linear analysis (paper I), in nonlinear situations (in paper II) and finally bringing here a new way to check accuracy and validity of full kinetic global codes in reduced geometry.

To end, we discussed our vortex-merging results in comparison of inverse-type cascade and of the concept of phase mixing. Here, the origin of the electric turbulence can be identified by considering the dynamics of particles located on the central stream, a diagnosis available by the multistream approach. The obtained results indicate that, in the inverse-cascade style process, which may follow the initial vortex-merging events, the waves are not in any sense locked to the original purely magnetic BGK waves. Indeed the pairwise vortex merging scenario represents a downward chirp in wave number rather than a wider spectrum implied in concepts such as "inverse cascade." Perhaps more sophisticated spectral diagnosis for larger and "open" systems could elucidate this behaviour in future work. Once the phase space resonance vortices are considerably melded, the behaviour during the evolution becomes that of more or less uncorrelated plasma structures. In other words, once the vortices cease to be well-connected with a pure magnetic trapping, the behaviour of the plasma becomes that of a plasma with a modified inhomogeneous distribution of hot electrons leading to the growth of an electrostatic field component.

The obtained sequences show clearly how quickly the system moves from a classic fully trapped well-organized plasma wave to a structure with much more phase space mixing. However, most of the thin filaments in phase space have disappeared over a long time due to the phase mixing. The problem is probably more crucial when relativistic effects are considered since such thin filaments can also be produced by Weibel-type instabilities. Thus, in the "Eulerian" or semiLagrangian approach, the numerical scheme must avoid the numerical instabilities driven by the reconstruction of $f$ and provides a "cleaning" of the details of the velocity space. As a result, finite-difference numerical simulation of the Vlasov equation usually do not suffer from the numerical instabilities driven by the filamentation in the velocity space. In both numerical Vlasov codes, the system approaches a stationary solution. This is due to finer and finer filamentation in phase space, which ultimately reaches the level of the mesh size. However, the relaxation towards a stationary state is "physical" and we have checked that it does not depend on the step of the grid.

A last point concerns the reversibility of the Vlasov equation. Of course, from a strictly mathematical point of view, the Vlasov equation is completely reversible. However, as we point out before, the classical phase space tends to become more and more intricated, so that the "cleaning" of thin filaments prevents the possibility of inverting the dynamics and thus recovering the initial condition. It must be pointed out that the semi-Lagrangian scheme is able to recover the collisionless linear Landau damping, which is essentially a phase mixing phenomenon. However in that case, the distribution function never loses memory of the initial data which is indeed reminiscent to the existence of a number of degrees of freedom in the Vlasov representation, which is virtually infinite. Thus it possible, in the case of the study of the linear Landau damping, to recover the initial state after the well-known recurrence time $T=\frac{2 \pi}{k \triangle v}$, where $k$ corresponds to the plasma wave number and $\Delta v$ is the standard size of the cell used in the velocity space. In the case of more complex situations, such as the study of plasma echoes, ${ }^{22}$ the "Vlasov" code, using the same semi-Lagrangian 
scheme, was found to be able to keep the information contained in the highly filamented distribution function until the time of the echo. As shown in Ref. 22, the echo is a typically ballistic effect and can be interpreted in terms of the partial reversibility properties of the physical system. On the other hand, because the treatment of the "streams" is exact in the multistream approach (in the sense that they constitute an exact class of solutions), the only error in the corresponding code is the round-off error due to the finite number of digits in the computer words in the $x-p_{x}$ phase space while the treatment is exact in $p_{y}$. Thus, the "multistream" code might allow a complete separation between the phenomena of numerical and physical irreversible process and to study the "interaction" between streams. The first one corresponds to a loss of information while the second involves a smoothing of informations by phase mixing, which, on the other hand, are still conserved for the computation of the evolution of the system.

\section{ACKNOWLEDGMENTS}

The author is indebted to the IDRIS computational center, Orsay, France for computer time allocation on their computers.

\footnotetext{
${ }^{1}$ A. Ghizzo and P. Bertrand, Phys. Plasmas 20, 082109 (2013).

${ }^{2}$ A. Ghizzo, Phys. Plasmas 20, 082110 (2013).

${ }^{3}$ R. L. Berger and R. C. Davidson, Phys. Fluids 15, 2327 (1972).

${ }^{4}$ A. I. Akhiezer and R. V. Polovin, Sov. Phys. JETP 3, 696 (1956); see also I. V. Smetanin, D. Farina, J. Koga, and S. V. Bulanov, Phys. Lett. A 320, 438 (2004).

${ }^{5}$ M. E. Innocenti, M. Lazar, S. Markidis, G. Lapenta, and S. Poedts, Phys. Plasmas 18, 052104 (2011).

${ }^{6}$ H. H. Kaang, C. M. Ryu, and P. H. Yoon, Phys. Plasmas 16, 082103 (2009).

${ }^{7}$ L. Palodhi, F. Califano, and F. Pegoraro, Plasma Phys. Controlled Fusion 51, 125006 (2009).

${ }^{8}$ L. Palodhi, F. Califano, and F. Pegoraro, Journal of Physics: Conference Series 208, 012075 (2010).

${ }^{9}$ A. Inglebert, A. Ghizzo, T. Reveille, P. Bertrand, and F. Califano, Phys. Plasmas 19, 122109 (2012).

${ }^{10}$ M. L. Begue, A. Ghizzo, and P. Bertrand, J. Comput. Phys. 151, 458 (1999).

${ }^{11}$ A. Ghizzo, F. Huot, and P. Bertrand, J. Comput. Phys. 186, 47 (2003).
}

${ }^{12}$ F. Huot, A. Ghizzo, P. Bertrand, E. Sonnendrücker, and O. Coulaud, J. Comput. Phys. 185, 512 (2003).

${ }^{13}$ Although the two philosophies PIC and Vlasov are different (we solve the Vlasov equation instead of solving the motion of individual particles), the numerical methods are somewhat similar. For instance for the 1D problem say, for a $(x, v)$ phase space; the distribution function, at time t, writes $f(x$, $v, t)$ and $f$ is advanced in time performing successive shifts either in the $x$-space (free particle motion) or in the $v$-space (acceleration related to the electric field). This is similar to the leap-frog scheme to advance particles in a PIC code. A "Vlasov" code needs a total number $N_{x} N_{v}$ points in phase space. In a PIC code we have also to consider $N_{x}$ cells of length $\Delta x$ to compute the field; $N_{v}$ can be compared as the number of particles in a cell. Nevertheless, $N_{v}$ cannot be small in a Vlasov code because of the phase space filamentation: typically $N_{v} \geq 50-100$. See Ref. 21 for more details.

${ }^{14}$ B. D. Fried, Phys. Fluids 2, 337 (1959).

${ }^{15}$ A. Stockem, M. E. Dieckmann, and R. Schlickeiser, Plasma Phys. Controlled Fusion 51, 075014 (2009).

${ }^{16}$ R. C. Davidson, D. A. Hammer, I. Haber, and C. E. Wagner, Phys. Fluids 15, 317 (1972).

${ }^{17}$ The smearing of the phase space structure is usually associated to the increase of the entropy. The Vlasov equation solution cannot change along a characteristic and often the characteristics mix together phase space regions where the distribution $f$ exhibits significant different values and then steep gradients are generated in $f$. In simulation that are done on a grid in phase space, the grid almost inevitably becomes too coarse as the fine graining develops. After a long time, the filaments have disappeared because of phase mixing. However, the trapping structure persists as a stationary solution and the entropy is then conserved in this time interval. To test the code, different phase space samplings have been used for the problem of the nonlinear Landau damping in the 1D case. As time increases, the entropy reaches a maximum value at about the same time that the instability saturates and then shows no tendency for further growth. A detailed examination of the time evolution shows that it takes somewhat larger to obtain the "cleaning" of the microstructure by increasing the phase space sampling. However, all simulation tests, used here, exhibit, after the "cleaning" the same state corresponding to values of entropy very close in the asymptotic plasma behaviour.

${ }^{18}$ R. Schlickeiser, Phys. Plasmas 11, 5532 (2004).

${ }^{19}$ U. Schaefer-Rolffs, I. Lerche, and R. Schlickeiser, Phys. Plasmas 13, 012107 (2006).

${ }^{20}$ T. Y. B. Yang, J. Arons, and A. B. Langdon, Phys. Plasmas 1, 3059 (1994).

${ }^{21}$ P. Bertrand, A. Ghizzo, M. R. Feix, E. Fijalkow, P. Mineau, N. D. Suh, and M. Shoucri, in Proceedings of the International Workshop on Linear Phenomena in Vlasov Plasmas, Cargèse, France 11-16 July 1088, edited by F. Doveil (Editions de Physique d'Orsay, 1989); see also A. Ghizzo, B. Izrar, P. Bertrand, E. Fijalkow, M. R. Feix, and M. Shoucri, Phys. Fluids 31, 72 (1988).

${ }^{22}$ G. Manfredi and M. R. Feix, Phys. Rev. E 53, 6460 (1996). 